\title{
Taller de Arquitectura: confluencia de ejes temáticos de innovación docente
}

\author{
Casares Gallego, Amparo; Raya de Blas, Antonio \\ Escuela Técnica Superior de Arquitectura, Universidade da Coruña.
}

\section{RESUMEN}

El Taller de Arquitectura aparece por la necesidad de integración de las distintas competencias que deberá adquirir el estudiantado durante el aprendizaje en el desarrollo de una propuesta arquitectónica relevante, que le ha de acercar a la realidad interdisciplinar que supone el trabajo profesional arquitectónico a través de una intervención concreta.

Para eso se tendrán en cuenta los conceptos de responsabilidad social y adaptación ambiental desarrollando siempre la capacidad crítica y el compromiso ético con la sociedad y las condiciones de confort actuales. Se pretende que el alumnado aborde un proceso complejo de desarrollo de una propuesta de intervención arquitectónica completa, desde su diseño hasta su realización técnica precisa, que abarque las fases de toma de datos, interpretación, elaboración y ejecución.

Se utiliza una confluencia de ejes temáticos de innovación docente que han de ajustarse a un plan de trabajo establecido en un cronograma que fije fases para garantizar la elaboración del proyecto completo en el período docente establecido.

Esta comunicación se centra principalmente en la exposición y valoración de las metodologías activas utilizadas, sus combinaciones y los resultados obtenidos. Las metodologías se analizan en relación con el resto de los ejes temáticos y su importante clave temporal.

PALABRAS CLAVE: Taller de Arquitectura, metodologías aprendizaje, docencia innovadora 


\section{CITA RECOMENDADA:}

Casares Gallego, A.; Raya de Blas, A. (2019): Taller de Arquitectura: confluencia de ejes temáticos de innovación docente. En De la Torre Fernández, E. (ed.) (2019). Contextos universitarios transformadores: construíndo espazos de aprendizaxe. III Xornadas de Innovación Docente. Cufie. Universidade da Coruña. A Coruña (pág.161-174).

DOI capítulo: https://doi.org/10.17979/spudc.9788497497121.161

DOl libro: https://doi.org/10.17979/spudc.9788497497121

\section{ABSTRACT}

The Architecture Workshop arises from the need to integrate the different skills that students must acquire during their learning in the development of a relevant architectural proposal, which must bring them closer to the interdisciplinary reality of professional architectural work through a specific intervention.

To this end, the concepts of social responsibility and environmental adaptation will be considered, always developing critical capacity and ethical commitment to society and current comfort conditions. The aim is for students to tackle a complex process of developing a complete architectural intervention proposal, from its design to its precise technical realisation, covering the phases of data collection, interpretation, elaboration and execution.

It uses a confluence of thematic axes of teaching innovation that have to conform to a work plan established in a schedule that sets phases to ensure the development of the complete project in the established teaching period.

This communication focuses mainly on the presentation and assessment of the active methodologies used, their combinations and the results obtained. The methodologies are analysed in relation to the rest of the thematic axes and their important temporal key.

KEY WORDS: Architecture workshop, learning methodologies, innovative teaching 


\section{INTRODUCCIÓN}

El Taller de Arquitectura aparece por la necesidad de integrar las distintas competencias que deberá adquirir el estudiantado durante su aprendizaje en el desarrollo de una propuesta arquitectónica relevante, que le ha de acercar a la realidad interdisciplinar que supone el trabajo profesional arquitectónico a través de una intervención concreta.

La enseñanza de la arquitectura integra conceptos de contenido social y adaptación ambiental desarrollando siempre la capacidad crítica y el compromiso ético con la sociedad y las condiciones de confort actuales.

Se pretende que el alumnado aborde un proceso complejo de desarrollo de una propuesta de intervención arquitectónica completa, desde su diseño hasta su realización técnica precisa, que abarque las fases de toma de datos, interpretación, elaboración y ejecución.

Para eso deberá adquirir las aptitudes necesarias para entender e integrar la historia y teoría de la arquitectura a partir de referentes culturales y arquitectónicos en el ámbito de estudio, reconocer los sistemas urbanos, de periferia y rurales, tomando el soporte territorial como clave para la implantación de la propuesta, elaborar la definición programática y valorar y definir los sistemas estructurales y constructivos más adecuados con las tecnologías existentes. El proyecto propuesto ha de demostrar un proceso creativo en busca de innovación en cualquiera de los aspectos señalados.

El Taller de Arquitectura se enfrenta a una ardua tarea, resultando un importante campo de ensayo de metodologías activas de aprendizaje.

Las Tecnologías de la Información y la Comunicación (TIC): plataforma Moodle, portfolios digitales, redes sociales, software especializado educativo... son de uso ineludible para el desarrollo del proyecto arquitectónico, objetivo del Taller de Arquitectura.

Respecto a los temas tratados y los contenidos exigidos, trabaja en el eje temático del impulso de la responsabilidad social universitaria.

Se utiliza el portfolio de arquitectura como recurso para la evaluación del aprendizaje. 
Además, la necesaria evaluación del aprendizaje por competencias específicas y transversales de la profesión de arquitecto demanda nuevas metodologías que abordaremos más adelante. Obliga, además, al trabajo colaborativo entre los docentes de las distintas especialidades de la disciplina.

Se utiliza una confluencia de ejes temáticos de la innovación docente que ha de ajustarse a un plan de trabajo establecido en un cronograma que fije fases para garantizar la elaboración del proyecto completo en el período docente establecido.

Esta comunicación se centrará principalmente en la exposición y valoración de las metodologías activas utilizadas, sus combinaciones y los resultados obtenidos. Las metodologías se analizarán en relación con resto de ejes temáticos y la importante clave temporal.

\section{DESCRICIÓN DE LA EXPERIENCIA}

Existen Talleres de Arquitectura desde el tercer cuatrimestre de la titulación de Grado en Estudios de Arquitectura hasta la finalización del Máster en Arquitectura. Pondremos el foco en el Taller de Proyecto Fin de Carrera en el que se desarrolla el trabajo fin de máster que otorga la capacidad profesional. Cuenta con 30 ECTS, a los que corresponden 10 horas de docencia presencial por crédito. Posee una característica única: el profesorado del Taller establece el apto para que los trabajos sean presentados ante tribunal. Será el tribunal, tras la exposición pública, el que les ponga nota. El tribunal cuenta con un/a arquitecto/a de reconocido prestigio propuesto por el colegio profesional, según se establece en la Orden Ministerial EDU/2075/2010.

Este Taller lo componen ocho profesores/as con diversas dedicaciones: dos de proyectos, dos de construcción, uno/a de estructuras, uno/a de urbanismo, uno/a de composición y uno/a de instalaciones. Todos/as ellos/as comparten tiempo y espacio de exposiciones teóricas y clases interactivas con todo el alumnado, con flexibilidad en función de las horas asignadas a su área de conocimiento. 
El Taller, como señalamos, trabaja con una confluencia de ejes temáticos de innovación docente y se estructura temporalmente fijando las fases de desarrollo que permitirán alcanzar el objetivo de elaborar el proyecto completo en el período docente establecido.

\section{- Utilización de TICS:}

Además de la indispensable utilización de Moodle para organizar la asignatura, compartir información... la web de la Escuela Técnica Superior de Arquitectura complementa datos sobre actividades y conferencias que pueden ser de interés. El alumnado utiliza Onedrive y Dropbox para compartir sus investigaciones y trabajos.

Como software especializado educativo podemos señalar:

Plataformas GIS (Sistema de Información Geográfico), AutoCad, Revit, llustrator, Photoshop, Scketchup, Grasshopper, Rhinoceros, Cype Estructuras, Cype Mediciones, Cype Mep, herramientas Office $365 \ldots$ Los procesos BIM (Building Information Modeling) se están incorporando desde el cuarto cuatrimestre según el plan de estudios.

Es necesaria la utilización de al menos dos tercios de estos programas para realizar el proyecto completo.

Para la elaboración de maquetas, se recurre a las tecnologías de fabricación digital: impresión 3D, cortadora láser, fresadora de control numérico computarizado... que permiten digitalizar objetos en tres dimensiones y fabricarlos materialmente. Estos recursos se encuentran en OFab de la Escuela (ETSAC).

\section{- Impulso de la responsabilidad social universitaria:}

Los temas de los Proyectos Fin de Carrera son propuestos por el profesorado. Actualmente, curso 2018-19, se están desarrollando seis, de los que cuatro de ellos trabajan sobre destacados aspectos de responsabilidad social:

- Condominio urbano: unidades residenciales para personas solas. A Coruña

- Escuela primaria en la Comunidad de El Manchón. Departamento de Retalhuleu. Guatemala

- Cohousing para personas mayores en 0 Vilar, San Pedro de Nós 
- Rehabilitación del entorno de la Colegiata Santa María la Real del Sar para centro de integración de refugiados y desplazados. Santiago de Compostela

No siempre ha sido así. Creemos que en este campo se han dado las circunstancias para que el avance sea reseñable. Se había trabajado ya en temas de cooperación (2009-10), de apoyo a colectivos urbanos (2014-15) como el "Proxecto Cárcere" en su reivindicación de la recuperación de este espacio para uso de la ciudad y de violencia de género (2017-18), pero aisladamente.

También se puede detectar la preocupación por la sustentabilidad, transversal a todos los temas y, en determinados casos, perspectiva de género.

\section{- Metodologías activas:}

La enseñanza de proyectos arquitectónicos es realmente un aprendizaje basado en proyectos. Se trata de proyectos reales, vinculados a la vida y a las necesidades que se detectan, habitualmente en el entorno cercano. El alumnado está vinculado al problema que ha de resolver, está conectado con su realidad. Se visita el lugar, se observan los usos y costumbres, los problemas, las carencias... Se habla con la ciudadanía sobre sus deseos, sus opiniones y valoraciones para acercarse a la vida cotidiana real. La parte de análisis y acercamiento al proyecto se trabaja en grupo. El alumnado tiene capacidad de elección del tema de su proyecto para que adquiera un rol activo y se favorezca la motivación académica. La clase al revés (Flipped Classroom), modelo de trabajo experimental que invierte los espacios y tiempos de explicación y trabajo en el aula y fuera de ella, es utilizada con una inversión más: el/la estudiante elabora y expone los contenidos que el profesorado presentó brevemente a través de esquemas, imágenes, planos...

Se ha ensayado esta metodología activa combinada con estudio de casos. El/la docente prepara casos similares al proyecto a resolver y los expone brevemente con materiales sugerentes: fotografías, vídeos, esquemas, planos... Se centran con claridad los aspectos que han de desarrollar en la búsqueda los/as estudiantes. En la siguiente clase el alumnado expone sus hallazgos al resto de la clase. Al principio del cuatrimestre el trabajo y la exposición 
pública es en grupo, de dos o tres componentes, para que se sientan más seguros. Después, tanto la investigación como la exposición, son personales. El Proyecto Fin de Carrera, que obligatoriamente es un trabajo individual, ha de ser expuesto públicamente ante tribunal. Esta fórmula es muy adecuada para preparar al alumnado para este intenso momento

Design thinking, el pensamiento del diseño, es propiamente la metodología de desarrollo de un proyecto arquitectónico. Esta herramienta de diseño parte del estudio de las necesidades y de las tecnologías existentes apropiadas para establecer una estrategia de diseño. En el proyecto arquitectónico creemos que falta una componente esencial de partida, que ha de estudiarse también, para establecer una estrategia innovadora y exitosa. Se trata del lugar, entendido en sentido amplio, clima, topografía, cultura, memoria... Las fases del design thinking son perfectamente aplicables al desarrollo de un proyecto: empatiza, define, idea, prototipa y testea.

El método de aprendizaje basado en problemas se utiliza desglosando los distintos aspectos que influyen en el proyecto: clima, topografía, vegetación, estructura de la propiedad, cultura, historia... que son estudiados y expuestos por el alumnado en grupos pequeños.

\section{- Recursos para la evaluación de los aprendizajes}

La necesaria evaluación del aprendizaje por competencias nos genera un cambio conceptual, que nos lleva a entender la evaluación como una fase más del aprendizaje ${ }^{1}$, La evaluación del Proyecto Fin de Carrera $(\mathrm{PFC}=\mathrm{TFM})^{2}$ del Máster en Arquitectura conducente a la profesión de arquitecto parte de no pocas limitaciones externas:

- La Directiva 2013/55/UE del Parlamento Europeo y del Consejo, al tratarse de una de las cinco profesiones reguladas.

\footnotetext{
${ }^{1}$ Fernandez March, A. (2010) desarrolla en su artículo esta concepción de la evaluación orientada al aprendizaje.

2 Zamora Polo, F. \& Sánchez Martín, J. (2015). Los autores describen el paso de muchas titulaciones la modelo existente en Arquitectura e Ingeniería y, concretamente, el riesgo de la conversión de un PFC en TFG. En este caso la conversión, no automática, del PFC ha sido al TFM del Máster profesionalizante.
} 
- Acceso independiente, para cada Escuela de Arquitectura del espacio europeo, al registro de reconocimiento de titulaciones del IMI (Sistema de Información del Mercado Interior).

- El Libro Blanco de la arquitectura.

- La Orden EDU 2075/2010 sobre las competencias del PFC, de 30 ECTs en proyectos de ejecución y 15 ECTS en proyecto básico, con docencia en Taller establece:

"Elaboración, presentación y defensa, una vez obtenidos todos los créditos de grado y master, de un ejercicio original realizado individualmente, ante un tribunal universitario en el que deberá incluirse al menos un profesional de reconocido prestigio propuesto por las organizaciones profesionales. El ejercicio consistirá en un proyecto integral de arquitectura de naturaleza profesional en el que se sinteticen todas las competencias adquiridas en la carrera, desarrollado hasta el punto de demostrar suficiencia para determinar la completa ejecución de las obras de edificación sobre las que verse, con cumplimiento de la reglamentación técnica y administrativa aplicable."

Así, la estructura de TFM, sus contenidos mínimos, su desarrollo, su docencia en Taller y las materias que lo constituyen están definidos por un complejo corseé generado por superposición de normativas.

A esta complejidad, se le une la experiencia obtenida en la extinta titulación de Arquitecto, donde se llegó a cifras de más de 600 estudiantes matriculados en la Escuela de A Coruña en PFC. Distinguir en este momento el TFG, perteneciente al Grado en Estudios en Arquitectura, sin atribuciones profesionales, con las complejidades inherentes a la escritura académica sobre arte y diseño ${ }^{3}$.

\footnotetext{
${ }^{3}$ Melles, G. \& Lockheart, J. (2.012). Los autores ponen de manifiesto las deficiencias de la escritura académica en el caso del arte y diseño. El TFG aparece como un texto académico en las Escuelas de Arquitectura, con larga tradición en el Proyecto de Fin de Carrera (PFC=TFM) de carácter profesional, pero no en los textos académicos de investigación o ensayo.
} 
En atención a lo expuesto, la estructura el PFC queda ordenado en dos partes diferenciadas, con necesarios ajustes en los sistemas de docencia y evaluación: el Proyecto Básico y el Proyecto de ejecución (adaptados a los contenidos del Código Técnico de la Edificación CTE). Sobre estas fases diferenciadas o hitos, pero en constante ajuste entre ambas, se emite evaluación colegiada argumentada (apto - no apto) por el profesorado del Taller. El apto permite pasar a la siguiente fase (Proyectos de ejecución y, finalmente, evaluación del Tribunal).

Además, el Reglamento específico de PFC para la Escuela de Arquitectura establece dos niveles diferenciados para la evaluación final del PFC: el apto del Taller que permite exponer el PFC ante el Tribunal y la calificación final de este que califica de aprobado a matrícula de honor.

Con todo esto, se ha optado por una evaluación continua del Taller, que utiliza la exposición pública de las ideas del estudiante plasmadas en el portafolios, como base de partida de la docencia, donde el profesorado facilita las oportunidades para el aprendizaje personalizado de cada estudiante. Los dicentes son capaces de desarrollar sus propuestas, pero, también, aprenden del resto de compañero en estas exposiciones públicas. Incluso los docentes tienen un necesario periodo de ajuste y contraposición de criterios, algo muy necesario.

Para ello, se implantan diversas metodologías, ya expuestas, con una serie de impases constantes (recopilación y exposición de información de forma colaborativa, maquetas, dibujos, videos, exposiciones, entregas parciales). Se acentúa la apuesta por procesos colaborativos y exposiciones públicas durante el Proyecto Básico, para trabajar progresivamente con una metodología de tablero en el desarrollo del Proyecto de Ejecución.

Estos dos hitos, legalmente establecidos (Proyecto básico y Proyecto de ejecución), se califican mediante rúbricas anidadas, estructuradas de forma similar, pero con tres criterios iniciales excluyentes:

- integridad documental, según norma, para la cual se ha creado una lista de control comentada por parte de la ETSAC para facilitar la labor del estudiantado. 
- evaluación continua del seguimiento del curso por los estudiantes

- errores graves (seguridad a incendios, estructural, de uso y acceso)

La estructura de rubricas anidadas permite descender a una justificación detallada ponderada por las áreas que participan en el Taller (urbanismo, proyectos, composición, construcción, estructuras e instalaciones).

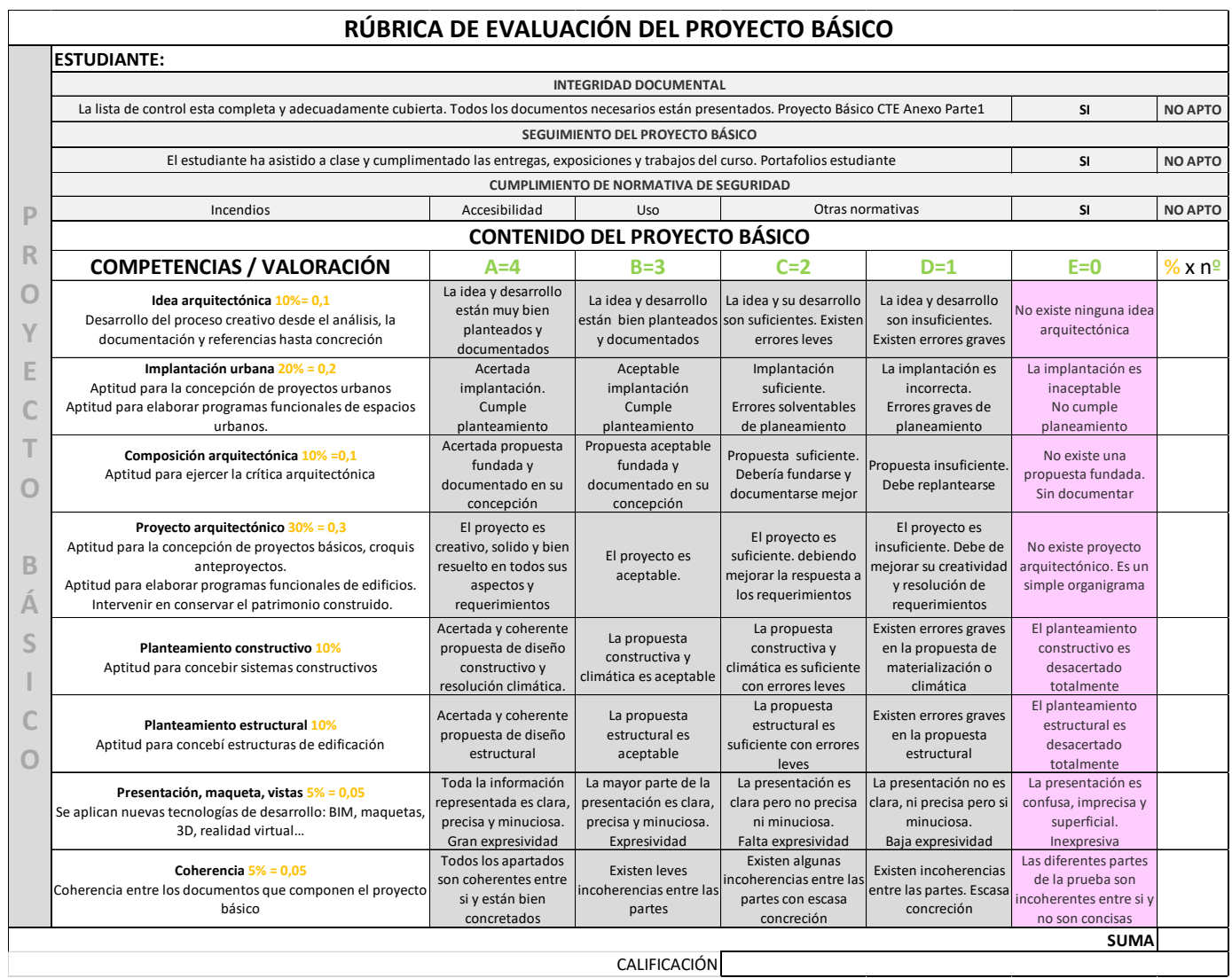

Tabla 1. Rúbrica de evaluación del proyecto básico. Elaboración propia. 


\begin{tabular}{|c|c|c|c|c|c|c|c|}
\hline \multicolumn{8}{|c|}{ RUBRICA DE EVALUACIÓN DEL PROYECTO DE EJECUCIÓN } \\
\hline \multirow{5}{*}{ P } & \multicolumn{7}{|l|}{ ESTUDIANTE: } \\
\hline & \multicolumn{7}{|c|}{ INTEGRIDAD DOCUMENTAL } \\
\hline & \multirow{2}{*}{\multicolumn{7}{|c|}{$\begin{array}{l}\text { La lista de control esta completa y adecuadamente cubierta. Todos los documentos necesarios están presentados } \\
\text { SEGUIMIENTO DEL PROYECTO DE EJECUCIÓN }\end{array}$}} \\
\hline & El estudiante ha asistido a clase y cumplime & entado las entregas, exp & osiciones y trabajos del & curso. Portafolios estudi & liante & & NO APTO \\
\hline & & CUMPLIMIEN & TO DE NORMATIVA DE S & SEGURIDAD & & & \\
\hline $\mathbf{R}$ & Incendios & Accesibilidad & Uso & Estructuras & Otras normativas & SI & NO APTO \\
\hline $\mathrm{n}$ & \multicolumn{7}{|c|}{ CONTENIDO DEL PROYECTO EJECUCIÓN } \\
\hline 0 & competencias / valoración & $A=4$ & $B=3$ & $C=2$ & $\mathrm{D}=1$ & $E=0$ & $\% \mathbf{x} \cap \stackrel{0}{2}$ \\
\hline E & \begin{tabular}{|c|} 
Desarrollo urbanístico e infraestructuras $10 \%$ \\
Aptitud para la práctica y el desarrollo de proyectos urbanos. \\
Capacidad para redactar y gestionar planes urbanisticos a \\
cualquier escala. Solución de ajardinamiento, \\
infraestructuras urbanas, equipamiento y pavimentos.
\end{tabular} & \begin{tabular}{|c|}
$\begin{array}{c}\text { Las soluciones son } \\
\text { acertadas } \\
\text { desarrollando los } \\
\text { valores expuesto en el } \\
\text { básico }\end{array}$ \\
\end{tabular} & $\begin{array}{l}\text { Las soluciones son } \\
\text { acertadas pero falta } \\
\text { desarrollo }\end{array}$ & $\begin{array}{c}\text { La propuesta es } \\
\text { aceptable aunque con } \\
\text { errores leves }\end{array}$ & $\begin{array}{c}\text { La propuesta esta mal } \\
\text { resuelta y tiene errores } \\
\text { graves }\end{array}$ & $\begin{array}{l}\text { No existe una } \\
\text { propuesta o esta no } \\
\text { cumple con la } \\
\text { normativa }\end{array}$ & \\
\hline $\begin{array}{l}\mathrm{T} \\
\mathrm{O}\end{array}$ & $\begin{array}{c}\text { Proyecto arquitectónico } 25 \% \\
\text { Aptitud para la concepción, la práctica y el desarrollo de: } \\
\text { proyectos de ejecución y dirección de obras. } \\
\text { Intervenir en restaurar y rehabilitar el patrimonio } \\
\text { construido. }\end{array}$ & $\begin{array}{c}\text { El proyecto de } \\
\text { ejecución ha sido } \\
\text { desarrollado } \\
\text { coherentemente con } \\
\text { la idea }\end{array}$ & $\begin{array}{c}\text { El propuesta resuelve } \\
\text { los aspectos }\end{array}$ & & $\begin{array}{c}\text { El proyecto es un } \\
\text { simple organigrama } \\
\text { sin concepción teórica }\end{array}$ & $\begin{array}{l}\text { No existe proyecto } \\
\text { arquitectónico. }\end{array}$ & \\
\hline \multirow{5}{*}{$\begin{array}{l}E \\
J \\
E \\
C \\
U \\
C \\
I \\
0 \\
N\end{array}$} & \begin{tabular}{|c|} 
Desarrollo constructivo $20 \%$ \\
Aptitud para concebir, calcular, diseñar, ejecutar e integrar \\
en edificios y conjuntos urbanos: Sistemas de división \\
interior, carpintería, escaleras y demás obra acabada; \\
Sistemas de cerramiento, cubierta y demás obra gruesa \\
\end{tabular} & $\begin{array}{c}\text { El desarrollo } \\
\text { constructivo es } \\
\text { acertado y coherente } \\
\text { con el proyecto básico }\end{array}$ & $\begin{array}{c}\text { El desarrollo } \\
\text { constructivo es } \\
\text { aceptable y coherente } \\
\text { con el proyecto básico }\end{array}$ & $\begin{array}{c}\text { El desarrollo } \\
\text { constructivo es } \\
\text { suficiente con errores } \\
\text { leves }\end{array}$ & $\begin{array}{c}\text { Existen errores graves } \\
\text { en el desarrollo } \\
\text { constructivo }\end{array}$ & $\begin{array}{c}\text { No existe desarrollo } \\
\text { constructivo } \\
\text { coherente. No cumple } \\
\text { normativa }\end{array}$ & \\
\hline & \begin{tabular}{|c|} 
Desarrollo estructural $20 \%$ \\
Aptitud para concebir, calcular, diseñar, ejecutar e integrar \\
en edificios y conjuntos urbanos, estructuras de edificación
\end{tabular} & \begin{tabular}{|c|} 
El desarrollo \\
estructural es acertado \\
y coherente con el \\
proyecto básico
\end{tabular} & $\begin{array}{c}\text { El desarrollo } \\
\text { estructural es } \\
\text { aceptable y coherente } \\
\text { con el proyecto básico } \\
\end{array}$ & \begin{tabular}{|c|}
$\begin{array}{c}\text { El desarrollo } \\
\text { estructural es } \\
\text { suficiente con errores } \\
\text { leves }\end{array}$ \\
\end{tabular} & $\begin{array}{c}\text { Existen errores graves } \\
\text { en el desarrollo } \\
\text { estructural }\end{array}$ & $\begin{array}{c}\text { No existe desarrollo } \\
\text { estructural coherente. } \\
\text { No cumple normativa }\end{array}$ & \\
\hline & \begin{tabular}{|c|} 
Desarrollo instalaciones $10 \%$ \\
Aptitud para concebir, calcular, diseñar, ejecutar e integrar \\
en edificios y conjuntos urbanos: instalaciones de suministro \\
y evacuación de aguas, electricidad, calefacción y \\
climatización
\end{tabular} & $\begin{array}{c}\text { El desarrollo de las } \\
\text { instalaciones es } \\
\text { acertado y coherente } \\
\text { con el proyecto básico }\end{array}$ & $\begin{array}{c}\text { El desarrollo de las } \\
\text { instalaciones es } \\
\text { aceptable y coherente } \\
\text { con el proyecto básico }\end{array}$ & $\begin{array}{c}\text { El desarrollo de las } \\
\text { instalaciones es } \\
\text { suficiente con errores } \\
\text { leves }\end{array}$ & $\begin{array}{c}\text { Existen errores graves } \\
\text { en el desarrollo de las } \\
\text { instalaciones }\end{array}$ & $\begin{array}{c}\text { No existe desarrollo } \\
\text { coherente de las } \\
\text { instalaciones. } \\
\text { No cumple normativa }\end{array}$ & \\
\hline & \begin{tabular}{|c|} 
Presentación, maqueta, vistas $5 \%$ \\
Se aplican nuevas tecnologías de desarrollo: BIM, maquetas, \\
3D, realidad virtual... \\
\end{tabular} & \begin{tabular}{|c|} 
Toda la información \\
representada es clara, \\
precisa y minuciosa. \\
Gran expresividad
\end{tabular} & $\begin{array}{c}\text { La mayor parte de la } \\
\text { presentación es clara, } \\
\text { precisa y minuciosa. } \\
\text { Expresividad }\end{array}$ & \begin{tabular}{|c|} 
La presentación es \\
clara pero no precisa \\
ni minuciosa. \\
Falta expresividad \\
\end{tabular} & $\begin{array}{c}\text { La presentación no es } \\
\text { clara, ni precisa pero si } \\
\text { minuciosa. } \\
\text { Baja expresividad }\end{array}$ & $\begin{array}{l}\text { La presentación es } \\
\text { confusa, imprecisa y } \\
\text { superficial. } \\
\text { Inexpresiva } \\
\end{array}$ & \\
\hline & $\begin{array}{c}\text { Coherencia } \\
5 \%\end{array}$ & $\begin{array}{c}\text { Todos los apartados } \\
\text { son coherentes entre } \\
\text { si y están bien } \\
\text { concretados }\end{array}$ & $\begin{array}{c}\begin{array}{c}\text { Existen leves } \\
\text { incoherencias entre las } \\
\text { partes }\end{array} \\
\end{array}$ & \begin{tabular}{|c|}
$\begin{array}{c}\text { Existen algunas } \\
\text { incoherencias entre las } \\
\text { partes con escasa } \\
\text { concreción }\end{array}$ \\
\end{tabular} & $\begin{array}{c}\begin{array}{c}\text { Existen incoherencias } \\
\text { entre las partes. Escasa } \\
\text { concreción }\end{array} \\
\end{array}$ & \begin{tabular}{|c}
$\begin{array}{c}\text { Las diferentes partes } \\
\text { de la prueba son } \\
\text { incoherentes entre si y } \\
\text { no son concisas }\end{array}$ \\
\end{tabular} & \\
\hline \multicolumn{8}{|c|}{ SUMA } \\
\hline & & & CALIFICACIÓN & & & & \\
\hline
\end{tabular}

Tabla 2. Rúbrica de evaluación del proyecto de ejecución. Elaboración propia. 


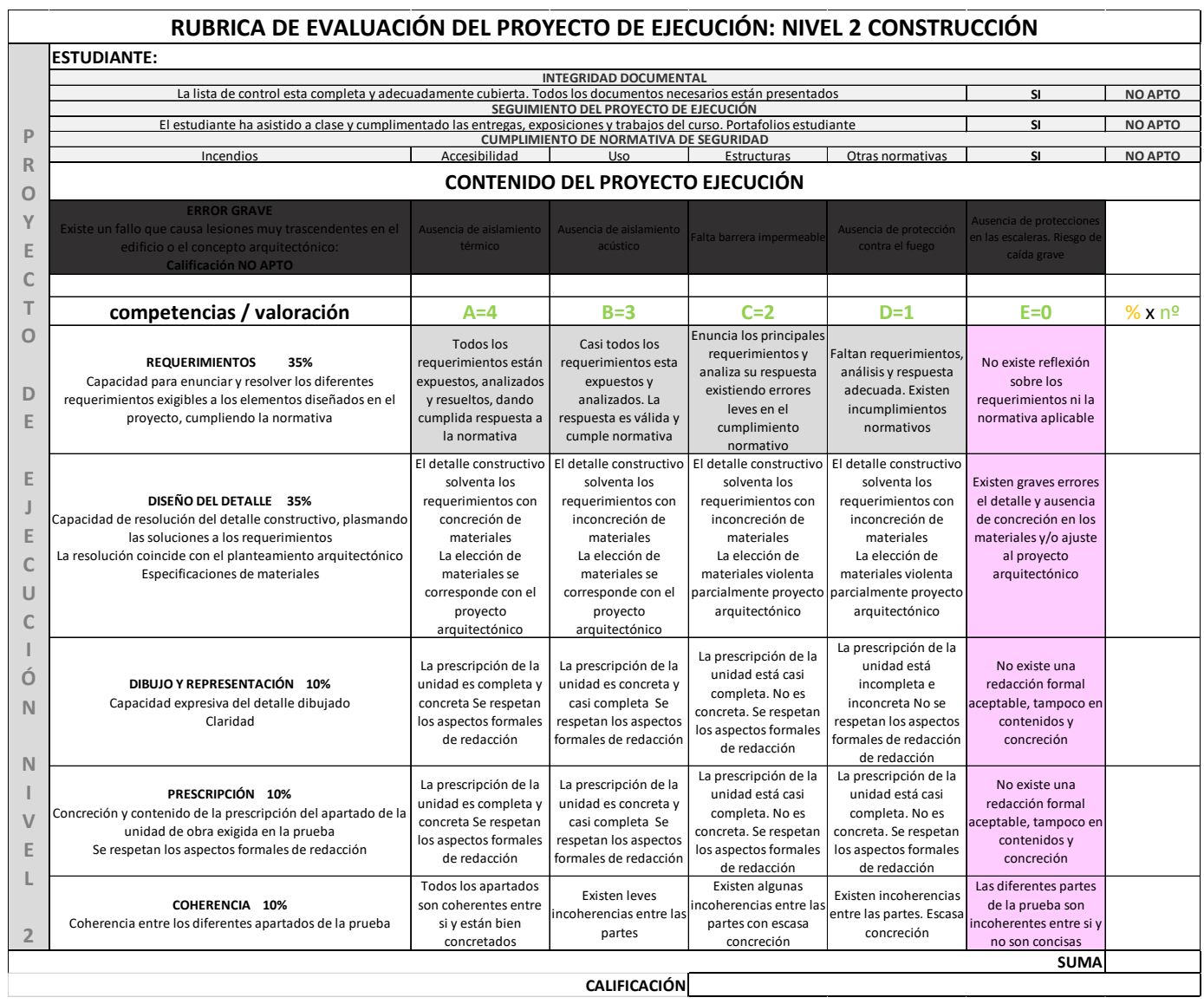

Tabla 3. Rúbrica de evaluación del proyecto de ejecución. Nivel 2 Construcción. Elaboración propia.

\section{- Colaboración entre docentes}

Una de las aportaciones más valiosas del Taller, cuando concurre, es la aparición de debate real y en profundidad sobre arquitectura, desde la diversidad disciplinar y personal. Concurre cuando la colaboración entre docentes está basada en el respeto y la curiosidad sobre las visiones ajenas, olvidando las paralizantes cuestiones de ego.

Creemos que es formativo para el alumnado participar en este enriquecedor entramado teórico y práctico en el que se producen discusiones y polémicas, siempre tranquila y elegantemente resueltas dentro de la pluralidad de criterios. 


\section{RESULTADOS}

La flipped classroom da unos magníficos resultados en más de un aspecto. Combinada con el estudio de casos centra al alumnado a analizar críticamente obras de otros arquitectos. El/la docente ayuda a estructurar la exposición: temas que se han de analizar, orden y tiempos. Más intensamente en la primera ocasión, que se realiza en grupo. Posteriormente la exposición es individual. El objetivo último es que expongan con competencia su trabajo personal, ante sus compañeros y el profesorado del taller, para que se encontren seguros/as y con recursos en la obligada defensa ante tribunal de su proyecto fin de carrera.

Design thinking explica el devenir de tiempos y actitudes del proceso creativo, dando una cierta tranquilidad a su enmarañado arranque. La multitud de posibilidades que presenta esta metodología la hace muy versátil. Comenzamos la fase de descubrimiento-empatía con tormentas de ideas, observación encubierta, investigación de referentes, mapa de contexto, consulta a expertos, análisis dafo, visita al lugar...

Cada año, cada taller trabaja en un tema diferente por lo que se ensayan las opciones que parecen más adecuadas.

\section{CONCLUSIONES}

El grupo de profesores/as, que llevamos cuatro años trabajando en este taller fin de carrera, procedentes de talleres de arquitectura de otros cursos, pensamos que es obligada la combinación de metodologías. Probablemente, en unos cursos más, habremos explorado en profundidad design thinking y podremos establecer pautas más precisas.

Si podemos apuntar ya que la flipped classroom en combinación con el estudio de casos y exposición por parte del alumnado del trabajo de investigación realizado es un buen comienzo para afianzar la empatía con el tema del proyecto. El resultado del método de aprendizaje basado en problemas es más formal, necesario, pero no acerca al alumnado al problema.

El método de aprendizaje basado en proyectos es ineludible. No podemos concebir otro planteamiento que no sea trabajar en proyectos auténticos y realistas, relacionados 
directamente con el contexto profesional y con impacto social, que mejoren la calidad de vida de las personas.

Como conclusión final, podemos establecer que el Taller de Arquitectura para el Proyecto Fin de Carrera es efectivamente un laboratorio de experimentación metodológica docente en activo, por lo que no parece que tenga un único resultado, ni final. Siempre estará en evolución, como la arquitectura, como la vida.

\section{REFERENCIAS}

Fernandez March, A. (2010). La evaluación orientada al aprendizaje en un modelo de formación por competencias en la educación universitaria. Revista de Docencia Universitaria, Vol.8 (n.1) 11-34 ISSN:1887-4592.

https://doi.org/10.4995/redu.2010.6216

Zamora Polo, F. \& Sánchez Martín, J. (2015) Los Trabajos Fin de Grado: una herramienta para el desarrollo de competencias transversales en la Educación Superior. REDU Revista de docencia Universitaria. Vol. 13 (3), Octubre - Diciembre 2015, 197-211 ISSN: 1887-4592 Melles, G. \& Lockheart, J. (2.012) Writing purposefully in art and design. Volume: 11 issue: 4 , page(s): 346-362. https://doi.org/10.1177/1474022211432116 